\title{
Magnitude of intracranial pressure changes following rigid external cervical collar immobilization in cases sustaining Traumatic Brain Injury: Impact analysis current status
}

\author{
Guru Dutta Satyarthee ${ }^{1}$, Luis Rafael Moscote-Salazar², Amit Agrawal ${ }^{3}$ \\ ${ }^{1}$ Department of Neurosurgery, Neurosciences Centre, AIIMS New Delhi, INDIA \\ ${ }^{2}$ Department of Neurosurgery, Critical Care Unit, RED LATINO, Latin American Trauma \& \\ Intensive Neuro-Care Organization, Bogota, COLOMBIA \\ ${ }^{3}$ Neurosurgery Department, Narayana Medical College \& Hospital, Nellore, INDIA
}

\begin{abstract}
Rigid collars are routinely used to immobilise the cervical spine during early phase of management of trauma cases until cervical clearance is obtained or else diagnosed as case of cervical spine injury following detailed clinical as well as neurological evaluation. Spinal injuries commonly coexist in patients sustaining severe head injury. Till date, there is no clear cut consensus about effect of application of a rigid collar in cases suffering with traumatic brain injury. However, concern are regularly raised over collar application, such practice may have adverse effect on cerebrovascular regulation and ultimately affecting intracranial pressure and outcome. Authors made detailed Pubmed, EMBASE, AMED, and Thomson Reuters, Medline line search and could find out six articles. According to existing literature, the cervical collar has the potential to influence intracranial pressure in patients suffering with head injury. There are several reports in the literature showing that cervical immobilization may alter intracranial pressure and the changes in ICP closely depend on the types of cervical collars used. Authors discuss the current status based on review of updated literature on possible effect on the intracranial pressure produced by application of rigid cervical collar and briefly literature is reviewed.
\end{abstract}

Key words: Utility of cervical collar, head injury, intracranial pressure, concern

\section{Introduction}

Poly-trauma cases carry high risk of cervical spine injury along with other associated injuries. These patients should be safely evacuated from the site of incident and common protocol includes immobilization of the cervical spine with application of a rigid external cervical collar to further prevent 
potential exaggeration of unstable cervical spine and associated spinal cord injuries in the prehospital settings (1). Placement of a protective cervical collar is common in cases of acute head trauma. Hard cervical collar or alternatives like rigid external cervical brace SOMI is applied and continued till the clearance cervical spine is obtained (1-4).

\section{Effect on intracranial pressure}

Mobbs et al. observed cases suffering with head trauma carry high risk of having a concomitant cervical spine injury and a rigid cervical collar is usually applied to each patient until spinal stability is confirmed. Hard collars application may cause potentially venous outflow obstruction and additionally represents as nocioceptive stimulus, may lead to intracranial pressure rise (5).

Mobbs et al. in their prospective study involving ten cases suffering with headinjury with a post- resuscitation Glasgow coma scale score of nine or less underwent measurement of intracranial pressure both the before and following the application of cervical hard collar. Authors observed 90\% cases had a rise in intracranial pressure following application of the collar and difference in pre- and post-application intracranial pressure was significant statistically. Mobbs et al. Concluded early cervical spine assessment and clearance in head-injured patients is recommended to minimize the risk of intracranial hypertension related to prolonged cervical spine immobilization with a hard collar (5).

Hunt et al. observed significant rise from the baseline intracranial pressure when the collars were applied with the mean rise of 4.6 $\mathrm{mmHg}$, which was statistically significant. Further. Mean rise was greater in patients with a baseline intracranial pressure greater than $15 \mathrm{mmHg}$ compared to counterpart having less than $15 \mathrm{mmHg}$. Authors observed no significant change in cardio-respiratory parameters during collar application, venous compression in the neck seems to be plausible explanation for intracranial pressure rise. Hunt et al. cautioned rigid collars should be removed as soon as cervical spine injury has been excluded in head-injured victims or else an alternative method of spinal stabilisation must be considered. (6)

Placing a cervical collar is a routine procedure and helps to reduce the risk of secondary spinal injury, however by altering the ICP it can lead to intracranial injury. According to these reports, the cervical collar has the potential to influence intracranial pressure in patients with traumatic brain injury. There are several reports in the literature showing that cervical immobilization may alter intracranial pressure and the changes in ICP closely depend on the types of cervical collars used. Effect of cervical collars on intracranial pressure in patients with head neurotrauma (7).

It has been shown that patients with severe head injury particularly those who are unstable, unconscious and intubated may have up to $14 \%$ of cervical lesions and up to $7 \%$ of these lesions $(5,8)$. The application of cervical collars has the potential risk to increase intracranial pressure causing obstruction to the venous drainage $(8,9)$. Other adverse effects of cervical immobilization are increased 
respiratory effort, skin ischemia, pain and discomfort

Kolb et al. prospectively evaluated the change in measured cerebrospinal fluid pressure after the application of a rigid Philadelphia collar in 20 adult patients without cervical spine injury undergoing lumbar puncture. The average pressure was $176.8 \mathrm{~mm} \mathrm{H} 2 \mathrm{O}$ initially and increased to an average of $201.5 \mathrm{~mm} \mathrm{H} 2 \mathrm{O}$ after collar placement (range 0 to 120 ) which statistically significant. Authors noted even small increment in pressure could be significant in patients who already have an elevated intracranial pressure (11).

Maissan et al. noted rigid cervical collar immobilization to the cervical spine are known to increase intracranial pressure in severe traumatic brain injury and the cerebral blood flow might decrease according to the Kellie Monroe doctrine. For this reason, the use of the collar in patients with severe traumatic brain injury has been abandoned from several trauma protocols in the Netherlands. Maissan et al in a prospective blinded cross-over study evaluated the effect of application of a rigid cervical collar in 45 healthy volunteers by measuring their optical nerve sheath diameter by transocular sonography an indirect non-invasive method to estimate ICP and pressure changes. They $\mathrm{He}$ studied healthy volunteers including 22 male and 23 female volunteers. They observed application of a collar resulted in a significant increase optic nerve sheath diameter in both side and concluded application of a rigid cervical collar significantly increases the optic nerve sheath diameter in healthy volunteers with intact cerebral autoregulation (12).

However, early of diagnosis of cervical spine injury will segregate those cases where cervical immobilization is not required anymore and cervical collar can be safely taken out and can be managed for associated traumatic brain injury (13-16).

However, current evidences have several limitations to generalize the findings, as analysis was primarily directed only to determine the effect of intracranial pressure rise on cervical collars and it does not pertain to neurological outcome of patients (7).

\section{Conclusion}

The currently available data fail to clearly establishing the impact of cervical collar application always produces rise in the intracranial pressure in those cases, who sustained traumatic head injury; although the rise in intracranially pressure may be of lesser impact than non- application might produced devastating consequences in form of worsening of unstable cervical spine injury as well as deterioration of neurological status and associated spinal cord injury. According to recent authors consensus an early evaluation of the cervical spine in patients with head trauma is necessary to reduce the impact of prolonged use of cervical collars and their possible influence on intracranial pressure, multicentric randomized case controlled studies are required to determine and actually quantify the exact and precise magnitude of rise in the intracranial pressure following immobilization of cervical spine with a rigid cervical collar. 


\section{Correspondence}

Dr. Guru Dutta Satyarthee

Associate Professor,

Department of Neurosurgery,

Room No. 714

Neurosciences Centre, AIIMS New Delhi

E-mail:duttaguru2002@yahoo.com

\section{References}

1. Satyarthee GD. Ways to Improve Outcomes of Traumatic Acute Spinal Cord Injury: Integrated Approaches of Improved Prehospital Care, the Adoption of Synergistic Medical and Surgical Intervention, Along with Care for Associated Systemic Injury and Rehabilitation and Social Inclusion. World Neurosurg. 2017; 101:786-787

2. Ho AMH, Fung KY, Joynt GM, Karmakar MK, Peng Z. Rigid cervical collar and intracranial pressure of patients with severe head injury. J Trauma 2002; 53(6): 1185-8.

3. Stone MB, Tubridy CM, Curran R. The effect of rigid cervical collars on internal jugular vein dimensions. Acad Emerg Med 2010; 17(1): 100- 2.

4. Lemyze M, Palud A, Favory R, Mathieu D. Unintentional strangulation by a cervical collar after attempted suicide by hanging. Emerg Med J 2011; 28(6): 532 .

5. Mobbs RJ, Stoodley MA, Fuller J. Effect of cervical hard collar on intracranial pressure after head injury. ANZ J Surg. 2002 Jun; 72(6):389-91.

6. Hunt K, Hallworth S, Smith M. The effects of rigid collar placement on intracranial and cerebral perfusion pressures. Anaesthesia. 2001; 56(6):511-3.

7. Moscote-Salazar LR, Godoy DA, Agrawal A, Rubiano AM. Effect of cervical collars on intracranial pressure in patients with head neurotrauma. Journal of Emergency Practice and Trauma 2017 . doi: 10.15171/jept.2017.03. 8. Davies G, Deakin C, Wilson A. The effect of a rigid collar on intracranial pressure. Injury 1996; 27(9): 647-9. 9. Kuhnigk H, Bomke S, Sefrin P. Effect of external cervical spine immobilization on intracranial pressure. Aktuelle Traumatol 1993; 23(8): 350-3. [In German].

10. Craig GR, Nielsen MS. Rigid cervical collars and intracranial pressure. Intensive Care Med 1991; 17(8): 504-5.

11. Kolb JC, Summers RL, Galli RL. Cervical collarinduced changes in intracranial pressure. Am J Emerg Med. 1999; 17(2):135-7.

12. Maissan IM, Ketelaars R, Vlottes B, Hoeks SE, den Hartog D, Stolker RJ. Increase in intracranial pressure by application of a rigid cervical collar: a pilot study in healthy volunteers. Eur J Emerg Med. 2017 Jul 19. doi: 10.1097/MEJ.0000000000000490

13. Verma SK, Singh PK, Agrawal D, Sinha S, Gupta D, Satyarthee GD, Sharma BS.

$\mathrm{O}$-arm with navigation versus $\mathrm{C}$-arm: a review of screw placement over 3 years at a major trauma center. $\mathrm{Br} \mathrm{J}$ Neurosurg. 2016 Dec;30(6):658-661.

14. Mishra A, Agrawal D, Gupta D, Sinha S, Satyarthee GD, Singh PK. Traumatic spondyloptosis: a series of 20 patients. J Neurosurg Spine. 2015 Jun;22(6):647-52.

15. Sawarkar DP, Singh PK, Siddique SA, Agrawal D, Satyarthee GD, Gupta DK, Sinha S, Kale SS, Sharma BS. Surgical management of odontoid fractures at level one trauma center: a single-center series of 142 cases. Neurol India. 2015 Jan-Feb;63(1):40-8.

16. Singh PK, Garg K, Sawarkar D, Agarwal D, Satyarthee GD, Gupta D, Sinha S, Kale SS, Sharma BS. Computed tomography-guided $\mathrm{C} 2$ pedicle screw placement for treatment of unstable hangman fractures. Spine (Phila Pa 1976). 2014 Aug 15;39(18):E1058-65 